\title{
Proposal of a Method to Measure Difficulty Level of Programming Code with Eye-Tracking
}

\author{
Tomoko Kashima $^{1}$, Shimpei Matsumoto ${ }^{2}$, and Shuichi Yamagishi ${ }^{2}$ \\ 1 Faculty of Engineering, Kinki University, \\ 1 Takaya Umenobe, Higashi-Hiroshima City, Hiroshima, 739-2116, Japan \\ 2 Faculty of Applied Information Science, Hiroshima Institute of Technology, \\ 2-1-1 Miyake, Saeki-ku, Hiroshima 731-5193, Japan
}

\begin{abstract}
In recent years, guaranteeing the educational quality is required in university education of Japan. With this situation in mind, we built study support environment with the information technology. As a result, we utilized the result for programming education and obtained the effect. There are various technical elements in the programming skill. However, many evaluations have adopted a comprehensive evaluation method. Therefore, a student's attainment to each technical element is indefinite. Some students become difficult to perform learning activities. So, in this research, programming notes the point which is the implicit thinking skill which is strongly related in study. Accumulation experience analyzes strongly related eye movement, and we aim at the standard construction for skill.
\end{abstract}

Keywords: programming, difficulty level, educational support, eyetracking.

\section{Introduction}

Recently, university education of Japan is demanded for the educational quality. Based on this situation, authors developed study training supporting environment using Information technology. Such learning environment has been utilized for the education of computer programming. We have some efficacy in those studies. However, there are various technical elements in programming skill. By such programming, the present condition is that a learner's evaluation is estimated by only overall points. Therefore, a learner is difficult to get to know the attainment level to each own technical element. So, in this study, we propose the method of presuming the difficulty of programming which applied the eye-tracking system [1] which is biological information.

Most investigations are classified with learner's analysis and discovery of the feature [2, teaching method proposal of programming instruction [3] and development of programming instruction support software 4. However, almost all study target a beginner. Most investigations are classified with learner's analysis and discovery of the feature, teaching method proposal of programming instruction and development of programming instruction support software. Almost all study targets a beginner. Usually, a beginner's study training speed differs.

M. Kurosu (Ed.): Human-Computer Interaction, Part II, HCII 2014, LNCS 8511, pp. 264-272, 2014.

(C) Springer International Publishing Switzerland 2014 
Therefore, it is necessary to change the study training supporting method and the teaching method according to the level of each learning. It is important to provide the various study training methods according to a learner's level.

The research task is divided into three phases in this study.First, we create a learner's evaluation index. Programming technique is subdivided and the skill standard for checking the learning situation of the technology assessment covering the many dimensions is developed. Skill items and those degrees of difficulty are built referred to item reaction theory, and existing previous study and books, and create a skill judging examination. Next, eye-tracking measurement experiment and its analysis are conducted. Measuring with a skill check is difficult for the process of the thinking according to the degree of experience. A learner's eye-tracking is measured and a learner's pattern of thinking is classified. A goal (predominance set) is set up using a Data Envelopment Analysis (DEA) model [5] by making into an input item the data obtained with the skill check, and the data obtained from biological information, and the teaching method according to a goal is proposed. A learner's skill improvement is supported by repeating these steps. Finally, we measure the learning efficiency according to each learner. And a learner's skill level and the carrer path which responded properly are shown. Here, the creation method of a concrete learner's evaluation index is explained. The technical element of programming is clarified and development of the learning materials for evaluation of the skill level of each element and a skill check table are developed. In order to learn the programming skill of a computer, a learner needs the ability of programming of not only the grammar of a programming language but an algorithm, or others. We clarify all skill elements.

Next, we develop the learning materials for checking the skill check about a learner's programming skill item. Learning materials are based on the structure which we developed until now. The structure takes up the module of the minimum unit. For example, the question of code complement form with which a starved area is compensated in an input, an output, and processing is used. It is the structure which can educate the thinking power according to the learner's technical element by the question. It feeds back to a learner by teaching and testing using the above structure. In the process in which this cycle is repeated, learner degree of comprehension and the degree of difficulty are quantitatively computed by item reaction theory. Based on a result, a learner group is defined according to a learner's achievement. Learning materials and a judgment examination are completed. A skill judging test applies the lesson module of Moodle, and collects learning histories efficiently.

It is easy to make a judgment mistaken for a correct answer in the proposed learning materials. However, even if a learner makes the same mistake, it is possible that the levels of a learner's understanding differ. Then, acquisition of programming skill pays attention to the point being strongly related in the logical thinking based on study training experience. This accumulation experience pays attention to the eye movement expressed strongly. By analyzing a learner's eyes, the process of consideration is clarified until it obtains the answer to each question. The degree of comprehension to experience and the component 
engineering which cannot be measured in a test is clarified by measuring a learner's eyes and classifying a learner's pattern of thinking. In this study, the analysis result of the eyes data especially done to various learners is introduced. The validity of the eyes data which is biological information as a method of measuring the degree of comprehension of program technology by that cause is described.

\section{Programming Education}

In the institution of higher education relevant to an information technology, programming skill is especially positioned as an important subject. Since the skill standard of programming is not fully defined, the contents of instruction differ for every organization. An attainment target, technical elements, and those setting levels are dependent on a teacher's educational philosophy in many cases. Student's results are decided by one-dimensional evaluation. In this case, mathematical logic thinking power is required strongly in many cases. The learner who makes this mathematical thinking power elated is not dependent on the teaching method or the contents, and good evaluation is obtained. On the other hand, other learners are evaluated by programming as a proper layer which is not. Historically, analysis of the achievement indicates that two layers certainly exist universally. This trend is not concerned with the difference of age, sex, and an academic level, but generating equally is known experientially. On many works, the presentation to a data input/output and a user is in the mainstream. It is rare to require advanced logic thinking power. A function, a class design, and the definition of a data structure are different technical elements from logic thinking power. The teacher should also evaluate these points. The improvement in software development power of Japan is indispensable in an international competition. Therefore, the learner whose learning evaluation of programming is not good should not judge that there is nothing properly. A learner is evaluated from many sides and it is thought that it is necessary to show the place of activity.

\subsection{Previous Study}

The study for programming is divided roughly into three kinds.

1. A learner's analysis and discovery of the feature

2. The teaching method proposal of programming instruction

3. Development of programming instruction support software

No. 1, the observation of an error pattern that a learner falls easily, the item and the coping-with method which bar a learner's understanding and the feature of the learner who makes programming unskillful. These are mostly in agreement in the report of previous study [6. However, many study has stopped at suggestion and a proposal for the beginner. Those study has proposed neither the study training supporting method for a beginner to advance a study training 
in maturity according to the level of each learning, nor the teaching method according to the level of a learner's learning.

No. 2, Hofuku et al. has perceived that there are many points which must be learned when studying programming, and has proposed the learning method which arranges a study training step in detail [7. However, they have not mentioned the point of preparing the directivity of various study trainings according to a learner's attainment level.

No. 3, It is considered as the support in a lecture, and the research tasks with main self-study learning environment construction. The LMS development based on Web by the study for self-study environmental construction is in use. The advantage of the study here is at the point which can feed back the result which may have had the use example of self-teaching environment analyzed to the teaching method [8]-10]. And, in order to aim at evoking interest, development of the function which paid its attention to the leisurely element, and presentation of an intuitive concept are done in many cases. The example of representation has Squeak, Alice, a pro grameen, and the Argo logic. As for the above, many results of research outstanding for the beginner have been reported. However, the support according to the achievement of the learner of the level which stepped up from the beginner is hardly tried in previous study. In order to be targeted at all the learner, it is necessary to formalize the step of the thinking according to programming experience. The trial into which skill of the technical element of programming introduced eye-tracking apparatus at this point depending on experience of coding for expression of experience is not checked as long as it is our investigation.

\section{Proposal}

In this study, we roughly divide a research task into the next three items, and are planning it.

\subsection{Making of Evaluation Index}

Programming technique is subdivided and the skill standard for checking the learning situation of the technology assessment covering the many dimensions is developed. Skill items and those degrees of difficulty are built referring to item reaction theory, and existing previous study and books, and create a skill judging test.

Specifically, a learner's evaluation index is created first. The technical element of programming is clarified and development of the learning materials for evaluation of the skill level of each element and a skill check table are developed. As shown in Table 1, the work which pulls out the item in connection with the ability of programming of not only the grammar of a programming language but an algorithm or others is done. Next, the learning materials for doing the skill check about a learner's programming skill item are developed. Learning materials are based on the structure developed by achievements [11. The module of 
the minimum unit is taken up, and it is a question of code complement form with which a starved area is compensated in an input, an output, and processing, and can educate the thinking power according to a technical element. It feeds back to a learner by teaching and testing using the above. In the process in which this cycle is repeated, learner degree of comprehension and the degree of difficulty are quantitatively computed by item reaction theory. Based on a result, a learner layer is defined according to a learner's achievement. Learning materials and a judgment test are completed by the above. A skill judging test utilizes the lesson module of Moodle, and collects learning histories efficiently.

\subsection{Eyes Measurement and Analysis}

About the process of the thinking according to the degree of experience which cannot be measured with a skill check, eyes are measured and a learner's patternof-thinking classification is done. A target (predominance set) is set up using a Data Envelopment Analysis (DEA) model by making these into an input item, and the teaching method according to a target is proposed.

It is easy to make a judgment mistaken for a correct answer in the learning materials done in the last fiscal year. However, while it is the same, it is possible that a level differs also in changing. Then, paying attention to a point strongly related in the logical thinking based on study training experience, this accumulation experience pays attention to acquisition of programming skill at the eye movement expressed strongly. By analyzing a learner's eyes, the process of consideration is clarified until it obtains the answer to each question. The degree of comprehension to experience and the component engineering which cannot be measured in a test becomes clear by measuring a learner's eyes and doing a learner's pattern-of-thinking classification. Fig. 1 shows the result of the eyes analysis experiment of the learner who carried out in advance of this study. The users 1 and 2 of Fig. 11 are learners who are insufficient of the skill which guesses the output of the program of Fig. 11.However, it becomes clear that it is an understanding level which is different when eyes are measured and analyzed. Although it turns out that he can understand the grammar used as the key to a program, and a value about the user 1, it is a difficult learner to arrive at an answer, without the ability to understand fine grammar. On the other hand, if it arrives at the contents which are not understood to some extent about the user 2 , it read over from the 1st line again and has repeated this operation. Thus, it becomes possible to read in an eye-tracking history the degree of comprehension which is not reflected in a result. The pattern of thinking of the learner who measured and got eyes is utilized for the teaching method making corresponding to the component engineering. It is assumed that the comment in question was the cause by which a learner layer with difficult follow continues existing in the point only depending on the explanation from a teacher's expert viewpoint traditional. Technique changes in the layer from which an experience level is different.

A gap exists explaining skillful work to a beginner. I think that the layer whose experience level of us is shallow will be what the technique of a somewhat 


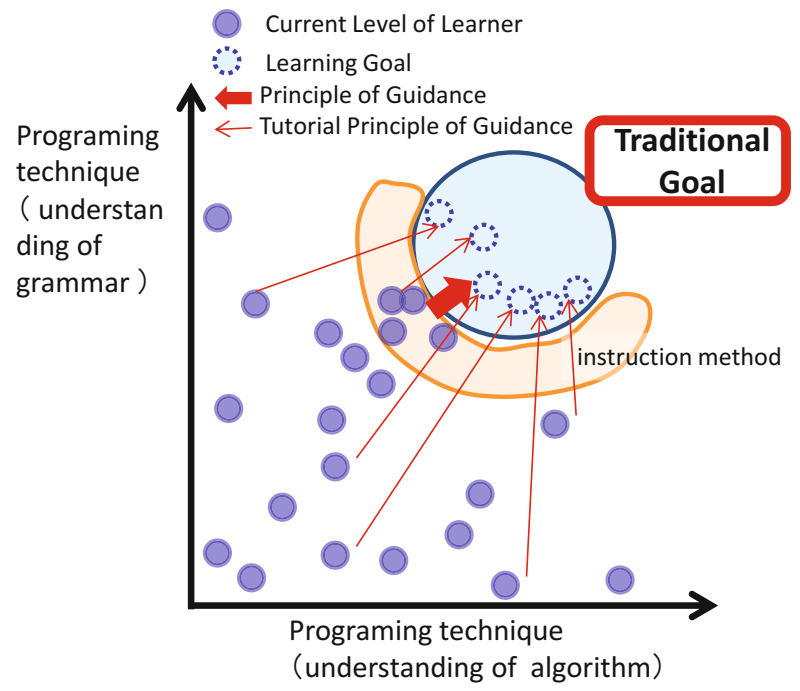

Fig. 1. The existing educational approach

high layer is imitated for (view place of eyes), and it will be important for it to promote step-up. With eyes analysis, the definition of learner layers is made strict and learner layers are simultaneously associated with a path. Grouping of the learner with each technologic-abilities difference is carried out based on the vector of many dimensions. A target (predominance set) is set up for every group, and it aims at proposing the teaching method according to a target. A Data Envelopment Analysis (DEA) model is used. Unlike the former, a setup of many targets is attained by this, and the target according to each learner's skill can be set up Fig. 2, In Fig. 2, three targets are set up and two or more teaching methods also exist. Thus, a setup of the target for developing the ability made elated for a learner is attained.

\subsection{Evaluation of Learning Efficiency Nature According to a Larner}

Here, a learner evaluates whether the study training is done efficiently. And, a learner's skill level and the carrer path which responded properly are shown. Specifically, each learner continues a study training toward a target in accordance with the teaching method. As a result, learning efficiency is evaluated according to the final place at which the learner arrived. Furthermore, matching with a learning stage and a career is done. As shown in Fig. 3, the check of the future target according to ability of a learner is attained, and he leads to the improvement in greediness for learning. And, in order to attain at a target, the check of the technical element which run short at present is attained, and becomes possible at any time about the directivity and the current position of a study training. 


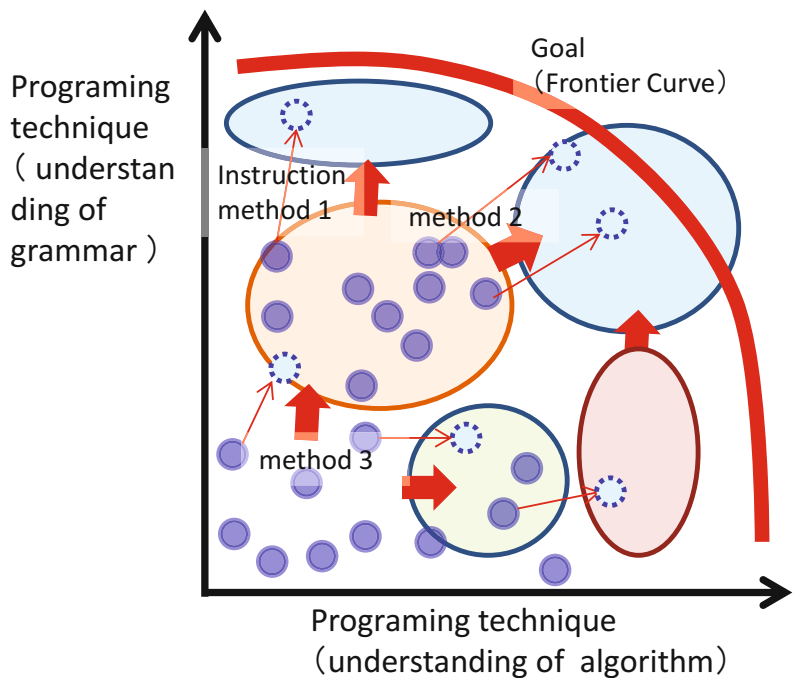

Fig. 2. The example by a Data Envelopment Analysis

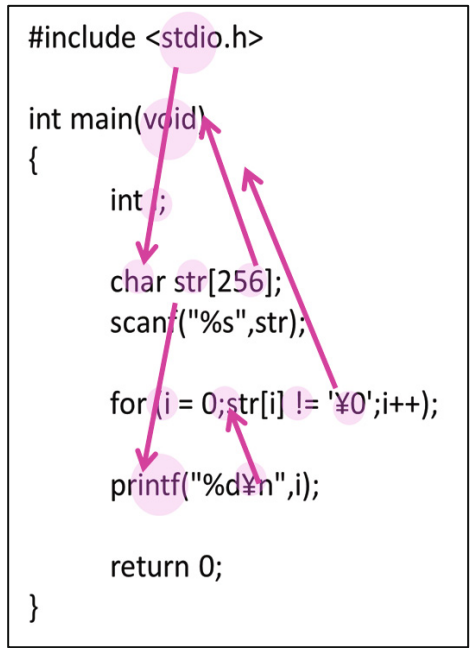

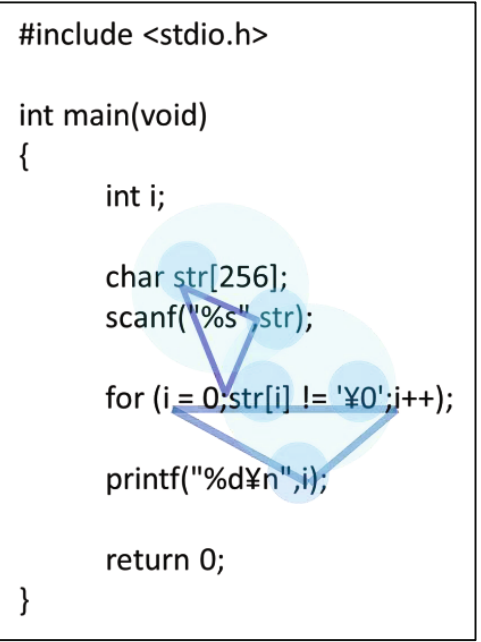

Fig. 3. Learner's eyes data 


\section{Result}

The proposal of a learner's evaluation index was first created with the proposal approach. As shown in Table 1, the technical item of programming was subdivided. It roughly divided with an understanding of grammar, and an understanding of the algorithm. About the detailed classification, knowledge needed for the $\mathrm{C}$ language which independent administrative agency Information-technology Promotion Agency shows was created to reference [8]. It becomes possible to also classify a learner according to these classifications. Next, eyes measurement experiment and analysis were conducted. It let the learner read a program and created two or more questions to which I get it to reply what kind of result to be displayed. The thinking process of the program was measured this time using the noncontact eyes measuring instrument. The eyes data shown in Fig. 3 is the learner who answered the inaccurate solution both.

However, it is shown by the learner from eyes data that degree of comprehension differs. Specifically, it turns out that the user 1 understands the line of the important key of a program. Although the data flow of the for sentence of a loop is also understood, not having led in the answer is shown. About the user 2 , he cannot understand the important point of a program, but it can observe signs that it rereads repeatedly to carry out one sentence and one-sentence understanding carefully repeatedly. Thus, it became clear that a learner's degree of comprehension and character can be read in eyes information according to a learner.

\section{Conclusion}

In this study, the thinking process of programming based on an eyes course was presumed for the purpose of making of the study training index which is useful for programming instruction. As a result, it became clear that it becomes possible to obtain the achievement level over the technical element according to the learner who was not evaluated until now. We would like to conduct many experiments, to propose the optimal career according to detailed skill investigation and skill, and to evaluate whether it is applicable in the future.

Acknowledgments. This work was partly supported by the Grant-in-Aid for Young Scientists (B-25750092), The Ministry of Education, Culture, Sports, Science and Technology, Japan.

\section{References}

1. Gog, T., Scheiter, K.: Eye Tracking as a Tool to Study and Enhance Multimedia Learning. Learning and Instruction 20(2), 95-99 (2010)

2. Lau, W., Yuen, A.: Modelling Programming Performance: Beyond the Influence of Learner Characteristics. Computers \& Education 57(1), 1202-1213 (2011) 
3. Kiss, G.: Teaching Programming in the Higher Education not for Engineering Students. Procedia - Social and Behavioral Sciences 103(26), 922-927 (2013)

4. Othman, M., Othman, M., Hussain, F.: Designing Prototype Model of an Online Collaborative Learning System for Introductory Computer Programming Course. Procedia - Social and Behavioral Sciences 90(10), 293-302 (2013)

5. Emrouznejad, A., Parkerb, B., Tavares, G.: Evaluation of Research in Efficiency and Productivity: A Survey and Analysis of the First 30 Years of Scholarly Literature in DEA. Socio-Economic Planning Sciences 42(3), 151-157 (2008)

6. Fushida, K., Tamada, H., Iguki, H., Fujiwara, K., Yoshida, N.: Coding Pattern Analysis for Novice Programmer in Programming Exercise, Technical Report of the Institute of Electronics, Information and Communication Engineers, Vol.111(481), SS2011-68, 67-72 (2012) (in Japanese)

7. Hofuku, Y., Cho, S., Nishida, T., Kanemune, S.: A Class Analysis by "De-Gapper": The Tool to Detect Gaps between Programs. Information Processing Society of Japan, SIG Notes 2013-CE-121(8), 1-6 (2013) (in Japanese)

8. Ogino, A., Tamada, H., Ueda, H.: Phynocation: A Prototyping of a Teaching Assistant Robot for C Language Class. In: Stephanidis, C. (ed.) Universal Access in HCI, Part IV, HCII 2011. LNCS, vol. 6768, pp. 597-604. Springer, Heidelberg (2011)

9. Tamada, H., Ogino, A., Ueda, H.: A Framework for Programming Process Measurement and Compiling Error Interpretation for Novice Programmers. In: Proc. of 2011 Joint Conference of the 21st International Workshop on and 6th International Conference on Software Process and Product Management, pp. 233-238 (2011)

10. Ishikawa, H., Maruyama, K., Terada, M.: Programming Study Support Using the Review Function of SNS. Proc. of Forum on Information Technology 11(3), 617-622 (2012) (in Japanese)

11. Ito, K., Sato, T., Tsubakimoto, M.: Analyzing Case Examples of a Self-Study Environment for Programing Education. Research Report of Japanese Society for Information and Systems in Education 27(4), 9-13 (2012) (in Japanese) 